\title{
Subacute thyroiditis - A rare cause of pyrexia of unknown origin
}

\author{
Balasubramaniam Branavan', Athwel Gamarallage Hemal Sugathapala² \\ ${ }^{1}$ Registrar, ${ }^{2}$ Senior Consultant Physician, Colombo South Teaching Hospital, Kalubowila, Sri Lanka. \\ Correspondence: Balasubramaniam Branavan, Registrar in Medicine, Colombo South Teaching \\ Hospital, Kalubowila, Sri Lanka \\ email address: hello.brana@live.com
}

\begin{abstract}
Pyrexia of unknown origin is a challenging problem to the physicians and rarely endocrine causes such as subacute thyroiditis can present as a pyrexia of unknown origin. It is usually a self-limiting condition and following post viral inflammatory process. It is common in young adults to middle age and does not involve in any autoimmune pathology. It has a self-limiting nature and symptomatic treatment is adequate. We report a 34 year old presented with a one month history of fever found to have high ESR and CRP with high free thyroxine level and treated as subacute thyroiditis with NSAID and she recovered completely from the acute illness.
\end{abstract}

\section{Introduction}

Pyrexia of unknown origin is a challenging problem to the physicians. Major causes could be categorized into infective, noninfective inflammatory, and neoplastic causes. Rarely endocrine disorders such as subacute thyroiditis can present as a pyrexia of unknown origin. It is a self-limiting post infective inflammatory condition and most of the time recover completely with a supportive measure.

\section{Case report}

We report a case of a 34 year old female presented to us with a history of fever for four weeks duration. She had daily fever spikes associated with lethargy and loss of weight. She denied any urinary symptoms, altered bowel habits, respiratory symptoms or vaginal discharge. She denied any recent travel history or significant contact history of tuberculosis.

On examination, she was febrile, pallor with small volume tender cervical lymph nodes. Thyroid gland was mildly enlarged and tender. Her pulse rate was $132 \mathrm{bpm}$ with a blood pressure of $120 / 80 \mathrm{mmHg}$ and rest of the cardiovascular examination was normal. Respiratory and abdominal examinations were unremarkable.

A full blood count showed white cell count of $14.6 \times 10^{6} / \mu \mathrm{L}$, haemoglobin of $9.4 \mathrm{~g} / \mathrm{dL}$ and platelet of $334 \times 10^{3} / \mu \mathrm{L}$. Blood film showed microchromic, microcystic cells with roulex formation. Erythrocyte sedimentation rate (ESR) was $108 \mathrm{~mm}$ in the $1^{\text {st }}$ hour with $C$ reactive protein of $30.7 \mathrm{mg} / \mathrm{dL}$. Liver and renal functions were within normal limits. Three blood cultures performed during fever spikes from different venipuncture sites were sterile. Viral screening for EBV, CMV, and toxoplasmosis antibodies were negative. Three samples of sputum AFB were negative and Mantoux reading was $4 \mathrm{~mm} .12$ lead electrocardiogram showed sinus tachycardia. transthoracic echocardiography did not show any valve abnormalities or any vegetations. Ultrasonography of abdomen does not show any focus of infection. Ultrasonography of neck showed heterogenically hypoechoic areas of both thyroid gland with reduced vascularity. Bilateral small volume reactive lymphnodes were also seen. $3^{\text {rd }}$ generation 
TSH value was less than $0.004 \mu \mathrm{lU} / \mathrm{mL}(0.4-4)$ and free $T_{4}$ value was $3.43 \mathrm{ng} / \mathrm{dL}(0.89-1.76)$. Antithyroid peroxidase (anti-TPO) antibodywas negative. Fine needle aspiration cytology did not perform due to patient's refusal. diclofenac sodium $50 \mathrm{mg}$ twice daily started following clinical diagnosis of subacute thyroiditis. Fever settled within 48hours and discharged from the ward. Diclofenac sodium was continued for 7 days. Follow up thyroid functions after six weeks revealed low normal TSH with normal free $\mathrm{T}_{4}$. He completely recovered from the illness. Follow up thyroid study was planned after six months and yet to be reviewed.

\section{Discussion}

Subacute thyroiditis, commonly referred to subacute granulomatous thyroiditis, also called as de quervain's thyroiditis is a self-limiting disorder and rare cause of hyperthyroidism. It is more common in females of young adulthood to middle age and incidence gradually reduced with advancing age. ${ }^{1}$ It is presumed to be caused by post viral inflammatory process. Thyroid autoimmunity does not play a role in subacute thyroiditis and antibodies are not present in majority of the cases.

Neck pain is the common presentation in subacute thyroiditis associated with constitutional symptoms such as malaise, fatigue, myalgia and anorexia. Symptoms of hyperthyroidism also can be noticed in these patients. Pyrexia of unknown origin is a rare presentation of subacute thyroiditis. ${ }^{2}$

Both thyroxine $\left(T_{4}\right)$ and triiodothyronine $\left(T_{3}\right)$ are elevated with suppressed thyroid stimulating hormone (TSH) level. Inflammatory markers such as ESR and CRP are usually elevated, occasionally ESR raised above $100 \mathrm{~mm} / 1^{\text {st }}$ hour as in our case. [3] Thyroid antibodies such as anti-thyroid peroxidase (anti-TPO) antibody and antithyroglobulin (anti-Tg) antibody are undetectable. Ultrasonography findings usually reveal heterogenous echogenicity of the thyroid gland with reduced doppler color flow in contrast to other causes of hyperthyroidism where increased flow noted. ${ }^{4}$ Low radio-iodine uptake with fail to improve uptake after TSH administration is also a characteristic feature in subacute thyroiditis.

Supportive treatment is adequate for subacute thyroiditis and antithyroid medications such as carbimazole are ineffective. Non-steroidal anti-inflammatory drugs (NSAIDs) such as ibuprofen, diclofenac sodium is effective in pain management and prednisolone used in refractory cases. It has an excellent prognosis and $10-20 \%$ of patient develop permanent thyroid dysfunction.

\section{Conclusion}

This case illustrates how subacute thyroiditis could be a cause for pyrexia of unknown origin. It is a self-limiting disorder and symptomatic treatment is adequate to achieve remission.

\section{References}

1. Fatourechi V, Aniszewski JP, Fatourechi GZ, et al. Clinical features and outcome of subacute thyroiditis in an incidence cohort: Olmsted County, Minnesota, study. J Clin Endocrinol Metab 2003; 88:2100.

2. Weiss BM, Hepburn MJ, Mong DP. Subacute thyroiditis manifesting as fever of unknown origin. South Med J 2000; 93:926-929.

3. Pearce EN, Bogazzi F, Martino E, et al. The prevalence of elevated serum C-reactive protein levels in inflammatory and noninflammatory thyroid disease. Thyroid 2003; 13:643.

4. Chaudhary V, Bano S. Thyroid ultrasound. Indian J EndocrMetab2013;17:219-27. 